\title{
Unique Occurrence of a Subependymoma Presenting Bilaterally with Hemorrhage: A Case Report
}

\author{
Yasmine Miguel $^{1}$, Gerard Jansen ${ }^{2}$, Fahad Alkherayf ${ }^{3}$ \\ ${ }^{1}$ Faculty of Medicine, University of Ottawa, Ottawa, Canada \\ ${ }^{2}$ Department of Pathology and Laboratory Medicine, The Ottawa Hospital, Ottawa, Canada \\ ${ }^{3}$ Department of Neurosurgery, The Ottawa Hospital, Ottawa, Canada \\ Email:ymigu017@uottawa.ca, gjansen@toh.on.ca, falkherayf@toh.on.ca
}

Received 28 December 2014; accepted 7 April 2015; published 14 April 2015

Copyright (C) 2015 by authors and Scientific Research Publishing Inc.

This work is licensed under the Creative Commons Attribution International License (CC BY). http://creativecommons.org/licenses/by/4.0/

(c) (i) Dpen Access

\begin{abstract}
Subependymomas are rare brain tumors derived from the ventricular system and are usually asymptomatic. The interest of this report is to demonstrate that subependymomas can very exceptionally present bilaterally and with hemorrhage. A 69-year-old woman presented with headache, ataxia, and focal neurologic deficits. Magnetic resonance imaging revealed a bilateral intra-axial lesion with unilateral hemorrhage. Patient underwent surgical resection of the tumor and the pathology was consistent with subependymoma. Postoperatively, patient recovered progressively with improvement of symptoms. This is the first case of a subependymoma that is both bilateral and hemorrhagic, two rare occurrences previously mutually exclusive in literature for this type of tumor. In the present case report we perform a review of the literature to analyze and compare retrospectively all other cases of hemorrhagic subependymomas.
\end{abstract}

\section{Keywords}

Brain Tumor Hemorrhage, Subependymoma, Bilateral Tumor, Lateral Ventricle

\section{Introduction}

Subependymomas are rare benign neoplasms of the brain. They typically originate from the subependymal cell plate. On histology, they correspond to World Health Organization Grade I tumors [1]. Accurate incidence is difficult to establish, as many remain asymptomatic until incidentally discovered on autopsy [2]. As a general estimate, subependymomas account for $0.2 \%$ to $0.7 \%$ of intracranial tumors [3] [4]. They are commonly encoun- 
tered along the walls of the ventricular system, located in $50 \%$ - $60 \%$ of cases in the fourth ventricle and in $30 \%$ - 40\% in the lateral ventricle [2] [5] [6]. They typically arise in middle-aged and elderly men [2] [7].

The natural history of subependymomas is characterized by a benign clinical course of indolent non-invasive growth, thus prognosis is generally favorable with infrequent post-treatment morbidity. Approximately $40 \%$ of quiescent subependymomas progress was slow growth and subsequently become symptomatic [1]. If they become symptomatic, clinical presentation is usually triggered by cerebrospinal fluid obstruction or hydrocephalus (e.g., headache, nausea, vomiting, gait ataxia, vertigo and dizziness) or by compression of neural structures (e.g., focal neurologic deficits) [1] [4] [7]. Spontaneous tumor-related hemorrhage represents an exceptionally rare and unusual event associated with this lesion [4] [8]. Only thirteen cases have been reported thus far [4]. This report presents this unique event of a bilateral supratentorial subependymoma with spontaneous hemorrhage. We also perform a review of the literature of hemorrhagic subependymomas.

\section{Case Report}

A 69-year-old lady presented with progressive headache, ataxia, apraxia, right-sided weakness and neglect. Her past medical history comprises hypertension, dyslipidemia, hypothyroidism and osteoarthritis. MRI (Figure 1) revealed large lobulated intraventricular masses involving the posterior body, occipital horn and trigone of both right and left ventricles with extension to the left corona radiata. The left lesion measured $25 \mathrm{~mm} \times 27 \mathrm{~mm} \times 37$ $\mathrm{mm}$ with intratumoral hemorrhagic transformation with extension to the parenchyma and significant perilesional vasogenic edema.

Patient underwent left parietal occipital craniotomy with excision of intraventricular tumor and neuro navigation and monitoring. The procedure was uncomplicated. Histopathology (Figure 2) demonstrated eosinophilicfibrillary matrix with sparsely cellular nuclei clustering with mild pleiomorphism (Figure 2A and Figure 2B), low proliferation index, small aggregates of siderophages (Figure 2B, arrow) and an intense positive GFAP reaction (Figure 2C), which are consistent with hemorrhagic subependymoma. Postoperative MRI confirmed removal of the tumor on the left side with extensive reduction of the edema and a stable appearance on the right side (Figure 3).

Postoperatively, the patient recovered progressively in the hospital with improvement in her right-sided weakness after rehabilitation. On her last follow-up, eight months later, her right-sided weakness and right-sided neglect resolved.

\section{Discussion}

Subependymomas are rare benign tumors of the central nervous system. They are most often found in the ventricular system rather than the spinal cord [1]. Bilateral disposition is an extremely rare finding with only three cases reported in the literature [7] [9] [10]. Their evolution is indolent and thus they don't typically trigger

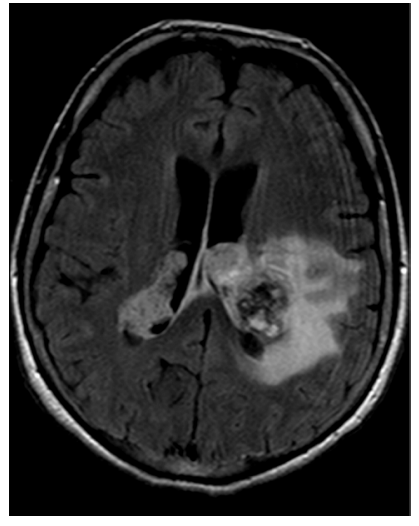

A

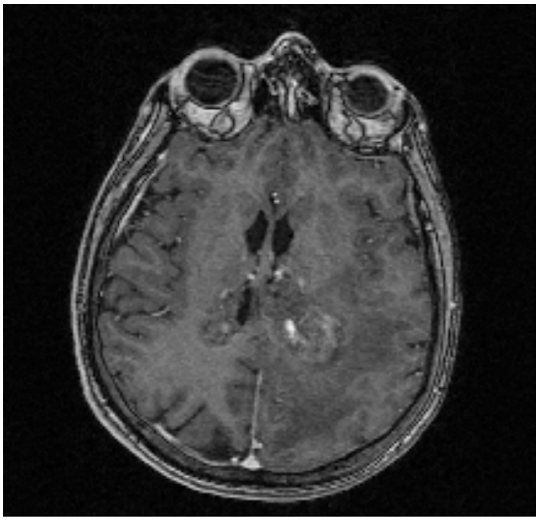

B

Figure 1. MRI views demonstrating bilateral cystic masses with parenchymal extension with no mass effect. A) Axial $T_{2}$ FLAIR sequence revealing bilateral hyperintense lesions and left intratumoral hemorrhage; B) Axial $\mathrm{T}_{1}$ sequence post gadolinium with no enhancement. 


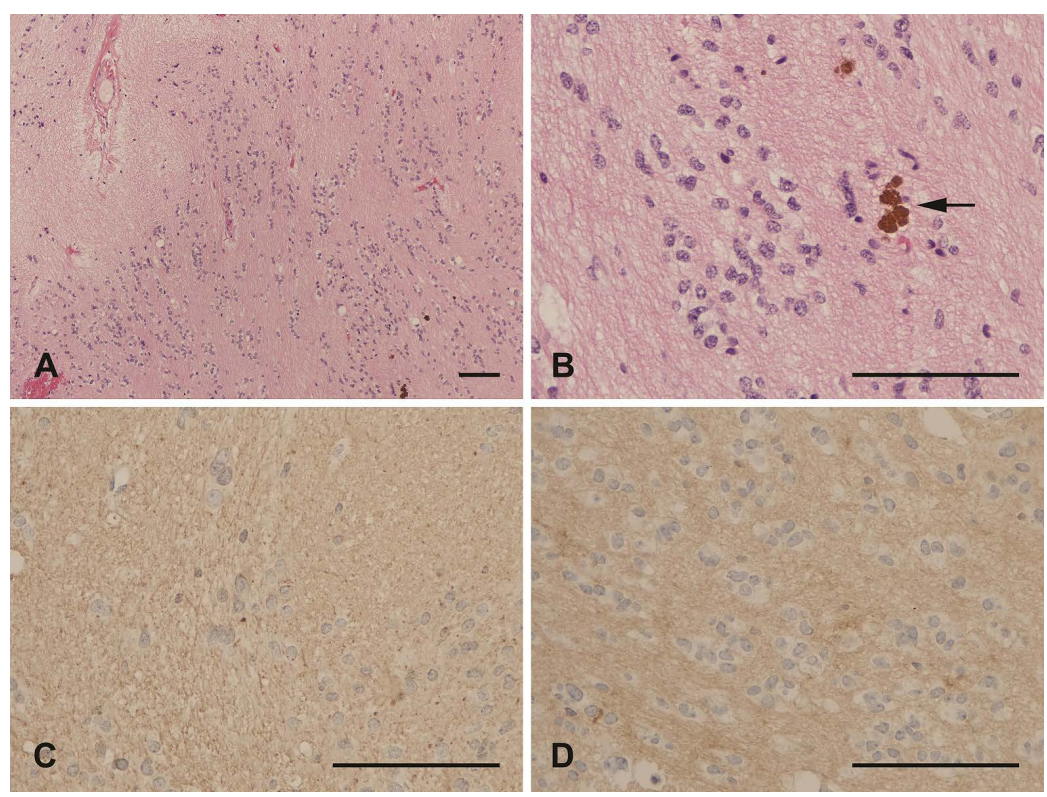

Figure 2. Histology of tumor. A) Low power (H \& E); B) High power (H \& E); C) Immunhistochemistry showing cell processes staining with GFAP; and D) showing cell membrane staining of cell processes with CD99. Bar in all images is 100 micrometer.

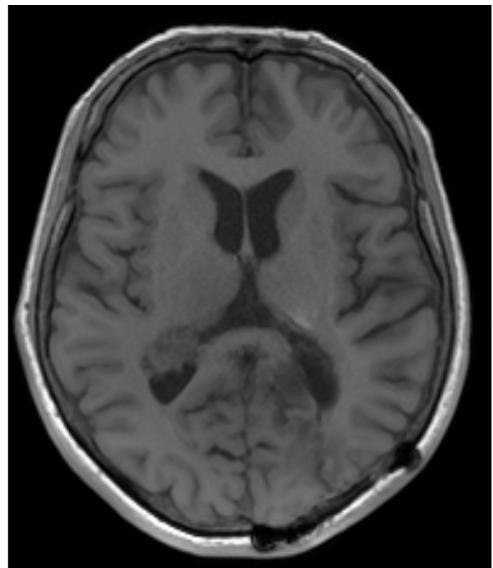

A

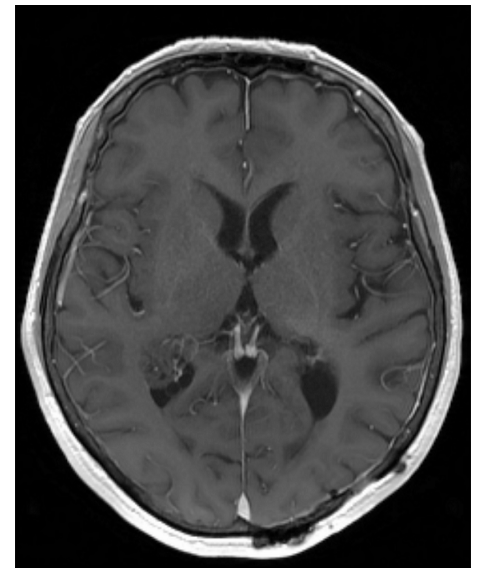

B

Figure 3. MRI sequences post surgery. A) Axial $T_{1}$ weighted MRI sequence revealing complete resection of left tumor with resolution of perilesional edema and hemorrhage; $\mathrm{B}) \mathrm{Axial} \mathrm{T}_{1}$ sequence post gadolinium with no enhancement.

symptoms or intracranial adverse events. These tumors are commonly smaller than $2 \mathrm{~cm}$ and greater sizes tend to produce symptoms associated with location [2] [4]. The exceptionality of this case lies in a symptomatic subependymoma presenting bilaterally with hemorrhagic transformation. It is the first reported case where two individually rare presentations of subependymomas occur together. Only thirteen cases of hemorrhagic subependymomas have been reported in the scientific literature thus far.

Usual radiologic characteristics of subependymomas involve well-defined solid and cystic intraventricular mass, T1 hypo to isointense and T2 hyperintense with little to no contrast enhancement, consistent with the findings of this patient [1] [3]-[5] [7]. Aggressive features such as peritumoral edema, mass effect, dense enhancement, high vascularity, calcification and intratumoral hemorrhage are rare with a prevalence of about $12 \%$ [1] [5] [8]. However, significant perilesional edema and hemorrhage are observed in this presented case.

Thirteen cases of hemorrhagic subependymomas are reported in the literature (Table 1). Three patterns of 
Table 1. Case reference of hemorrhagic subependymomas in the literature.

\begin{tabular}{|c|c|c|c|c|c|c|}
\hline $\begin{array}{l}\text { Case } \\
\text { No. }\end{array}$ & Reference & $\begin{array}{l}\text { Age/ } \\
\text { Gender }\end{array}$ & Clinical Presentation & $\begin{array}{l}\text { Location/ } \\
\text { Size }(\mathrm{cm})\end{array}$ & Risk Factors & Hemorrhage \\
\hline 1 & Scheithauer (1978) [2] & $81 / F$ & Headache, altered consciousness & LV/Large & NA & IT \\
\hline 2 & Changaris et al. (1981) [16] & 16/M & Headache, blurred vision & $\mathrm{AT}, \mathrm{OH} / 7$ & No & IT/SAH \\
\hline 3 & Seiki et al. (1984) [17] & $33 / \mathrm{F}$ & Headache, altered consciousness & AT/NA & No & IVH \\
\hline 4 & Yamasaki et al. (1990) [18] & $54 / \mathrm{F}$ & Headache & $\mathrm{FH} / 5$ & No & IT \\
\hline 5 & Marra et al. (1991) [19] & $42 / \mathrm{F}$ & Headache & $\mathrm{FH} / 2.5$ & NA & IVH, SAH \\
\hline 6 & DiLorenzo et al. (1991) [20] & $46 / \mathrm{M}$ & Headache & $\mathrm{FH} / 4$ & AHT & IVH, SAH \\
\hline 7 & Lindboe et al. (1992) [13] & $63 / M$ & Disorientation, memory loss & $\mathrm{FH} / 5$ & Vascularized tumor & IT, IVH, SAH \\
\hline 8 & Viale (1994) [21] & $52 / \mathrm{M}$ & Headache, altered consciousness & $\mathrm{FH} / 3$ & NA & IT \\
\hline 9 & Furie and Provenzale (1995) [22] & $46 / M$ & Headache & $\mathrm{LV} / 2$ & NA & IT \\
\hline 10 & Carrasco et al. (2010) [8] & $71 / \mathrm{M}$ & Altered consciousness & $\mathrm{FH} / 3$ & $\begin{array}{l}\text { AHT, Anticoagulant } \\
\text { therapy }\end{array}$ & IVH \\
\hline 11 & Akamatasu et al. (2010) [5] & $32 / \mathrm{M}$ & Headache, altered consciousness & $\mathrm{LV} / 2$ & No & IVH \\
\hline 12 & Sharma et al. (2010) [14] & $25 / M$ & Headache, blurred vision & LV, AT/NA & Vascularized tumor & IT \\
\hline 13 & Landriel et al. (2013) [4] & $32 / \mathrm{M}$ & Headache, posterior cervical pain & 4th $V / 3$ & No & ICM, SAH \\
\hline 14 & Present case & $69 / \mathrm{F}$ & Headache, focal neurologic deficits & LVB, PE/3.7 & AHT & IT, IP \\
\hline
\end{tabular}

AHT: arterial hypertension; AT: atrium; CR: coronal radiate; F: female; FH: frontal horn; ICM: intra cisterna magna; IP: intraparenchymal; IT: intratumoral; IVH: intraventricular hemorrhage; LV: lateral ventricle; LVB: Lateral ventricle (bilateral); M: male; NA: not available, OH: occipital horn; PE: parenchymal extension; SAH: subarachnoid hemorrhage; 4th V: fourth ventricle.

hemorrhage has been reported-extratumoral, intratumoral or mixed [8]. The pathophysiology of hemorrhage in subependymomas is unknown [1] [8] [11] [12]. However, Liu et al. [11] theorized that extratumoral hemorrhage was a consequence of tearing of surrounding veins from extended tumor growth while intratumoral hemorrhage resulted from rupture, endothelial proliferation, degeneration or thrombosis of abnormal fragile tumoral vessels in vascularized tumors [5] [8] [11]. However, there is no clear explanation of intratumoral hemorrhage given that subependymomas are typically avascular. Yet, some case reports [13] [14] suggest that there is vascular growth in subependymomas thus structural abnormalities in tumoral vessels may explain the origin of the bleed. As well, increased venous pressure associated with increased intracranial pressure or tumor expansion may trigger hemorrhage in these thin-walled tumoral vessels [15].

There are several risk factors that predispose to tumoral hemorrhage. On radiology, these include extended tumor growth, vascularized lesion and location where the tumor is prone to a traumatic mechanism such as below the foramen magnum [4] [8]. Clinically, these include vascular risk factors such as generalized hypertension, intracranial hypertension and anticoagulant therapy [4] [8]. The patient featured in this case report presented two important risk factors: hypertension and an unusual large subependymoma.

Clinical presentation of hemorrhagic subependymomas differs amongst the different types of hemorrhage. Extratumoral hemorrhage often seems indistinct from subarachnoid hemorrhage manifestation, while acute intratumoral bleeding can produce a sudden increase mass effect, generating acute obstruction of the cerebrospinal fluid pathways, and thus produce symptoms of hydrocephalus [8].

\section{Conclusion}

Subependymomas are extremely rare tumors commonly arising in the ventricular system of the brain. They are noninvasive and commonly follow an asymptomatic benign clinical course. Although unusual, subependymomas should be considered as a possible cause of cerebral hemorrhage in patients even without risk factors for intracranial hemorrhage. Spontaneous hemorrhage induced by subependymoma can be life threatening and justify neurosurgical intervention. 


\section{References}

[1] Wiestler, O.D. and Schiffer, D. (2000) Subependymoma. In: Kleihues, P. and Cavenee, W.K., Eds., Pathology and Genetics of Tumours of the Nervous System, International Agency for Research on Cancer (IARC) Press, Lyon, 80-81.

[2] Scheithauer, B.W. (1978) Symptomatic Subependymoma: Report of 21 Cases with Review of the Literature. Journal of Neurosurgery, 49, 689-696. http://dx.doi.org/10.3171/jns.1978.49.5.0689

[3] Jain, A., Amin, A.G., Jain, P., Burger, P., Jallo, G.I., Lim, M., et al. (2012) Subependymoma: Clinical Features and Surgical Outcomes. Neurological Research, 34, 677-684. http://dx.doi.org/10.1179/1743132812Y.0000000064

[4] Landriel, F., Besada, C., Migliaro, M., Christiansen, S., Goldschmidt, E., Yampolsky, C., et al. (2013) Atypical Hemorrhagic Presentation of a Fourth Ventricle Subependymoma: Case Report. Neurologia Medico-Chirurgica, 53, 828831. http://dx.doi.org/10.2176/nmc.cr2012-0292

[5] Akamatsu, Y., Utsunomiya, A., Suzuki, S., Endo, T., Suzuki, I., Nishimura, S., et al. (2010) Subependymoma in the Lateral Ventricle Manifesting as Intraventricular Hemorrhage-Case Report. Neurologia Medico-Chirurgica, 50, 1020-1023. http://dx.doi.org/10.2176/nmc.50.1020

[6] Nishio, S., Morioka, T., Mihara, F. and Fukui, M. (2000) Subependymoma of the Lateral Ventricles. Neurosurgical Review, 23, 98-103.

[7] Rath, T.J., Sundgren, P.C., Brahma, B., Lieberman, A.P., Chandler, W.F. and Gebarski, S.S. (2005) Massive Symptomatic Subependymoma of the Lateral Ventricles: Case Report and Review of the Literature. Neuroradiology, 47, 183188. http://dx.doi.org/10.1007/s00234-005-1342-3

[8] Carrasco, R., Pascual, J.M., Navas, M., Fraga, J., Manzanares-Soler, R. and Sola, R.G. (2010) Spontaneous Acute Hemorrhage within a Subependymoma of the Lateral Ventricle: Successful Emergent Surgical Removal through a Frontal Transcortical Approach. Neurocirugía, 21, 478-483. http://dx.doi.org/10.1016/S1130-1473(10)70100-7

[9] Kumar, R., Sarkari, A. and Kakkar, A. (2012) Mirror Image Subependymoma. Neurology India, 60, 684-685. http://dx.doi.org/10.4103/0028-3886.105228

[10] Rushing, E.J., Cooper, P.B., Quezado, M., Begnami, M., Crespo, A., Smirniotopoulos, J.G., Ecklund, J., Olsen, C. and Santi, M. (2007) Subependymoma Revisited: Clinicopathological Evaluation of 83 Cases. Journal of Neuro-Oncology, 85, 297-305. http://dx.doi.org/10.1007/s11060-007-9411-6

[11] Liu, Y.G., Liu, M., Zhu, S.G., Jiang, Y.Q., Li, G. and Li, X.G. (2002) Intracranial Tumouralhaemorrhage-A Report of 58 Cases. Journal of Clinical Neuroscience, 9, 637-639. http://dx.doi.org/10.1054/jocn.2002.1131

[12] Iwama, T., Ohkuma, A., Miwa, Y., Sugimoto, S., Itoh, T., Takada, M., et al. (1992) Brain Tumors Manifesting as Intracranial Hemorrhage. Neurologia Medico-Chirurgica, 32, 130-135. http://dx.doi.org/10.2176/nmc.32.130

[13] Lindboe, C.F., Stolt-Nielsen, A. and Dale, L.G. (1992) Hemorrhage in a Highly Vascularized Subependymoma of the Septum Pellucidum: Case Report. Neurosurgery, 31, 741-745. http://dx.doi.org/10.1227/00006123-199210000-00019

[14] Sharma, M., Velho, V., Ghodgaonkar, P. and Palande, D. (2010) Symptomatic Subependymoma of the Lateral Ventricle: A Rare Entity-A Case Report and Review of Literature. The Internet Journal of Neurosurgery, 7, 7.

[15] Nozaki, T., Sato, H., Yamazoe, T. and Namba, H. (2015) Diffuse Astrocytoma Initially Presenting as a Massive Intracerebral Hemorrhage: Case Report. Neurologia Medico-Chirurgica, 55, 86-88.

[16] Changaris, D.G., Powers, J.M., Perot Jr, P.L., Hungerford, G.D. and Neal, G.B. (1981) Subependymoma Presenting as Subarachnoid Hemorrhage: Case Report. Journal of Neurosurgery, 55, 643-645. http://dx.doi.org/10.3171/jns.1981.55.4.0643

[17] Seiki, Y., Terao, H., Shibata, I., Tsukahara, K., Tsutsumi, S. and Kudo, M. (1984) A Case of Subependymoma in the Lateral Ventricle with Intraventricular Hemorrhage. No Shinkei Geka. Neurological Surgery, 12, $761-765$ (in Japanese).

[18] Yamasaki, T., Kikuchi, H., Higashi, T., Yamabe, H. and Moritake, K. (1990) Two Surgically Cured Cases of Subependymoma with Emphasis on Magnetic Resonance Imaging. Surgical Neurology, 33, 329-335. http://dx.doi.org/10.1016/0090-3019(90)90201-Y

[19] Marra, A., Dario, A., Scamoni, C., Cerati, M., Crivelli, G. and Dorizzi, A. (1991) Intraventricular Subependymoma Presenting as Subarachnoid Hemorrhage. Case Report. Journal of Neurosurgical Science, 35, 213-215.

[20] DiLorenzo, N., Rizzo, A. and Ciappetta, P. (1991) Subependymoma of the Septum Pellucidum Presenting as Subarachnoid Hemorrhage. Neurochirurgia, 34, 125-126.

[21] Viale, G.L. (1994) Subependymomas of the Lateral Ventricles. British Journal of Neurosurgery, 8, 765-767.

[22] Furie, D.M. and Provenzale, J.M. (1995) Supratentorial Ependymomas and Subependymomas: CT and MR Appearance. Journal of Computer Assisted Tomography, 19, 518-526. http://dx.doi.org/10.1097/00004728-199507000-00002 